PROCEEDINGS OF THE

AMERICAN MATHEMATICAL SOCIETY

Volume 135, Number 2, February 2007, Pages 569-578

S 0002-9939(06)08740-5

Article electronically published on August 2, 2006

\title{
STRONG UNIQUE CONTINUATION FOR $m$-TH POWERS OF A LAPLACIAN OPERATOR WITH SINGULAR COEFFICIENTS
}

\author{
CHING-LUNG LIN
}

(Communicated by David S. Tartakoff)

\begin{abstract}
In this paper we prove strong unique continuation for $u$ satisfying an inequality of the form $\left|\triangle^{m} u\right| \leq f\left(x, u, D u, \cdots, D^{k} u\right)$, where $k$ is up to $[3 m / 2]$. This result gives an improvement of a work by Colombini and Grammatico (1999) in some sense. The proof of the main theorem is based on Carleman estimates with three-parameter weights $|x|^{2 \sigma_{1}}(\log |x|)^{2 \sigma_{2}} \exp \left(\frac{\beta}{2}(\log |x|)^{2}\right)$.
\end{abstract}

\section{INTRODUCTION}

Let $\Omega$ be a connected open subset of $\mathbb{R}^{n}(n \geq 2)$ containing 0 . In [6], Colombini and Grammatico prove some results of strong unique continuation for $u$ satisfying an inequality of the form

$$
\left|\triangle^{m} u\right| \leq f\left(x, u, D u, \cdots, D^{k} u\right) \quad \text { for } k=m .
$$

In this paper we are interested in $u$ satisfying the following forms:

$$
\left|\triangle^{m} u\right| \leq C_{0} \sum_{|\alpha| \leq m-1}|x|^{-2 m+|\alpha|}\left|D^{\alpha} u\right|+C_{0} \sum_{|\alpha|=m}^{[3 m / 2]}|x|^{-2 m+|\alpha|+\epsilon}\left|D^{\alpha} u\right|,
$$

where $0<\epsilon<1 / 2$ and the orders of lower order terms are up to [3m/2].

Theorem 1.1. Let $u \in H_{\text {loc }}^{2 m}(\Omega)$ be a solution of (1.2), and for all $N>0$ let

$$
\int_{|x| \leq R}|u|^{2} d x=O\left(R^{N}\right), \quad R \rightarrow 0 .
$$

Then $u$ is identically zero in $\Omega$.

This problem for $m=1$ has drawn a lot of attention in partial differential equations and mathematical physics. For the development of this problem, the author refers the readers to [15. In particular, their work was focused on second order equations in which the coefficients of the lower order terms are allowed to be singular (see [2], 3, 11] and [5]). Here we mention two articles which are closely related to our work in this paper.

Hörmander [8] proved that if $u \in H_{l o c}^{1}(\Omega)$ satisfies

$$
|\Delta u| \leq C_{1}|x|^{-2+\epsilon}|u|+C_{2}|x|^{-1+\epsilon}|\nabla u|, \quad \epsilon>0,
$$

and $u$ vanishes of infinite order at 0 , then $u$ is identically zero in $\Omega$.

Received by the editors August 23, 2005.

2000 Mathematics Subject Classification. Primary 54C40, 14E20; Secondary 46E25, $20 \mathrm{C} 20$.

The author was supported in part by the Taiwan National Science Council, NSC 93-2119-M194-007. 
Regbaoui [13] extended Hörmander's result to the sharp case $\epsilon=0$ with small $C_{2}$. His proof was also based on suitable Carleman estimates, but he worked with the strictly convex weights $\varphi_{\beta}(x)=\exp \left(\frac{\beta}{2}(\log |x|)^{2}\right)$ rather than the usual polynomial weights. As for negative results, some counterexamples were given when $C_{2}$ is not small (such as [1] and [16]).

For the case $m=2$, Borgne [4] got some results about strong unique continuation when $k=3$ in (1.1). On the other hand, Watanabe [14] established some uniqueness results for $m=3$ with $k=5$. For $m=4$, Ōkaji [10 solved unique continuation when $k=7$. For the higher orders, Protter [12] got the unique continuation for inequalities of the form (1.1) in which $k$ is up to [3m/2]. According to the counterexamples for $n=2$ in [7, Colombini and Grammatico [6] proved the sharp results about the coefficients of the $m$-th lower order terms for strong unique continuation with some cases when $k=m$. In this paper, we consider the cases (1.2).

We will follow Regbaoui's approach in [13] and organize this paper as follows. In Section 2, we shall study the asymptotic behavior of the function $u$ near 0 which guarantees the use of the singular weights $|x|^{2 \sigma_{1}}(\log |x|)^{2 \sigma_{2}} \varphi_{\beta}^{2}$. Some of the key Carleman estimates with the weights $|x|^{2 \sigma_{1}}(\log |x|)^{2 \sigma_{2}} \varphi_{\beta}^{2}$ will be derived in Section 3. Using these Carleman estimates, we prove Theorem 1.1 in Section 4.

\section{Carleman estimates With polynomial Weights}

Lemma 2.1. There exists a positive constant $C_{m}$ such that for any $u \in C_{0}^{\infty}\left(\mathbb{R}^{n} \backslash\{0\}\right)$ and for any $\tau \in\{k+1 / 2, k \in \aleph\}$, we have the estimate

$$
\sum_{|\alpha| \leq 2 m} \int \tau^{2 m-2|\alpha|}|x|^{-2 \tau+2|\alpha|-n}\left|D^{\alpha} u\right|^{2} d x \leq C_{m} \int|x|^{-2 \tau+4 m-n}\left|\triangle^{m} u\right|^{2} d x .
$$

Proof. In [13], Regbaoui proved in Lemma 2.1 for any $u \in C_{0}^{\infty}\left(\mathbb{R}^{n} \backslash\{0\}\right)$ and for any $\tau \in\{k+1 / 2, k \in \aleph\}$ that

$$
\sum_{|\alpha| \leq 2} \int \tau^{2-2|\alpha|}|x|^{-2 \tau+2|\alpha|-n}\left|D^{\alpha} u\right|^{2} d x \leq C \int|x|^{-2 \tau+4-n}|\triangle u|^{2} d x .
$$

By the repeated use of (2.2), we can get (2.1).

Remark 2.2. The estimate (2.1) in Lemma 2.1 remains valid if we assume $u \in$ $H_{l o c}^{2 m}(\Omega)$ with compact support and satisfying for all $|\alpha| \leq 2 m$ and all $N>0$, $\int_{|x| \leq R}\left|D^{\alpha} u\right|^{2} d x=O\left(R^{N}\right)$ as $R \rightarrow 0$. This can be easily obtained by cutting $u$ off for small $|x|$ and regularizing.

Motivated by 8 and [13, we get the following theorem. The proof of this theorem is based on Lemma 2.1. From now on, $c$ stands for a generic constant and its value may vary from line to line.

Theorem 2.3. Let $u \in H_{l o c}^{2 m}(\Omega)$ be a solution to (1.2). If $|\alpha| \leq 2 m$, then

$$
\int_{|x| \leq R}\left|D^{\alpha} u\right|^{2} d x=O\left(e^{-B R^{-\epsilon / m}}\right), \quad R \rightarrow 0,
$$

for some positive constant $B$. 
Proof. Following Hörmander's argument in [9] (Corollary 17.1.4., p. 8), we show that if $u \in H_{l o c}^{2 m}(\Omega)$ is a solution of (1.2), then for all $|\alpha| \leq 2 m$ and for all $N>0$

$$
\int_{|x| \leq R}\left|D^{\alpha} u\right|^{2} d x=O\left(R^{N}\right), \quad R \rightarrow 0 .
$$

In view of Remark 2.2, we can apply (2.1) to the function $\xi u$, where $\xi(x) \in$ $C_{0}^{\infty}\left(\mathbb{R}^{n}\right)$ such that $\xi(x)=1$ for $|x| \leq R$ and $\xi(x)=0$ for $|x| \geq 2 R(R>0$ sufficiently small). Here the number $R$ is not yet fixed and is given by $R=(\gamma \tau)^{-m / \epsilon}$, where $\gamma>0$ is a large constant which will be chosen later. Using the estimate (2.1) and the equation (1.2), we can derive that

$$
\begin{aligned}
& \sum_{|\alpha| \leq m-1} \tau^{2 m-2|\alpha|} \int_{|x| \leq R}|x|^{-2 \tau+2|\alpha|-n}\left|D^{\alpha} u\right|^{2} d x \\
& +\sum_{|\alpha|=m}^{2 m} \gamma^{2 m} \int_{|x| \leq R}|x|^{-2 \tau+2|\alpha|-n+2 \epsilon}\left|D^{\alpha} u\right|^{2} d x \\
& =\sum_{|\alpha| \leq m-1} \tau^{2 m-2|\alpha|} \int_{|x| \leq R}|x|^{-2 \tau+2|\alpha|-n}\left|D^{\alpha} u\right|^{2} d x \\
& +\sum_{|\alpha|=m}^{2 m} R^{-2 \epsilon} \tau^{-2 m} \int_{|x| \leq R}|x|^{-2 \tau+2|\alpha|-n+2 \epsilon}\left|D^{\alpha} u\right|^{2} d x \\
& \leq \sum_{|\alpha| \leq m-1} \tau^{2 m-2|\alpha|} \int_{|x| \leq R}|x|^{-2 \tau+2|\alpha|-n}\left|D^{\alpha} u\right|^{2} d x \\
& +\sum_{|\alpha|=m}^{2 m} \tau^{2 m-2|\alpha|} \int_{|x| \leq R}|x|^{-2 \tau+2|\alpha|-n}\left|D^{\alpha} u\right|^{2} d x \\
& \leq \sum_{|\alpha| \leq 2 m} \tau^{2 m-2|\alpha|} \int|x|^{-2 \tau+2|\alpha|-n}\left|D^{\alpha}(\chi u)\right|^{2} d x \\
& \leq C_{m} \int|x|^{-2 \tau+4 m-n}\left|\triangle^{m}(\chi u)\right|^{2} d x \\
& \leq c C_{m} \int_{|x| \leq R}|x|^{-2 \tau+4 m-n}\left|\triangle^{m} u\right|^{2} d x \\
& +c C_{m} \int_{|x|>R}|x|^{-2 \tau+4 m-n}\left|\left[\chi, \triangle^{m}\right] u\right|^{2} d x \\
& \leq c C_{m} C_{0} \sum_{|\alpha| \leq m-1} \int_{|x| \leq R}|x|^{-2 \tau+2|\alpha|-n}\left|D^{\alpha} u\right|^{2} d x \\
& +c C_{m} C_{0} \sum_{|\alpha|=m}^{[3 m / 2]} \int_{|x| \leq R}|x|^{-2 \tau+2|\alpha|-n+2 \epsilon}\left|D^{\alpha} u\right|^{2} d x \\
& +c C_{m} \int_{|x|>R}|x|^{-2 \tau+4 m-n}\left|\left[\chi, \triangle^{m}\right] u\right|^{2} d x,
\end{aligned}
$$

where $[\cdot, \cdot]$ denotes the commutator.

Therefore, carefully checking terms on both sides of (2.5), we can choose $\gamma$ and let $\tau$ be large enough such that all terms with $\int_{|x| \leq R}|x|^{-2 \tau+2|\alpha|-n}\left|D^{\alpha} u\right|^{2} d x$ and 
$\int_{|x| \leq R}|x|^{-2 \tau+2|\alpha|-n+2 \epsilon}\left|D^{\alpha} u\right|^{2} d x$ on the right side of (2.5) are absorbed by the lefthand side. We now fix such $\gamma$. By construction of $\chi$ we have $\left|D^{\alpha} \chi\right| \leq c_{1} R^{-|\alpha|}$, where $c_{1}$ is a positive constant. Consequently, it follows for $R<1 / 2$ and (2.5) that

$$
\begin{aligned}
& \tau^{-2 m}(R / 2)^{-2 \tau+4 m-n+2 \epsilon} \sum_{|\alpha| \leq 2 m} \int_{|x| \leq R / 2}\left|D^{\alpha} u\right|^{2} d x \\
\leq & \sum_{|\alpha| \leq 2 m} \tau^{2 m-2|\alpha|}(R / 2)^{-2 \tau+2|\alpha|-n+2 \epsilon} \int_{|x| \leq R / 2}\left|D^{\alpha} u\right|^{2} d x \\
\leq & \sum_{|\alpha| \leq 2 m} \tau^{2 m-2|\alpha|} \int_{|x| \leq R / 2}|x|^{-2 \tau+2|\alpha|-n+2 \epsilon}\left|D^{\alpha} u\right|^{2} d x \\
\leq & \sum_{|\alpha| \leq m-1} \tau^{2 m-2|\alpha|} \int_{|x| \leq R}|x|^{-2 \tau+2|\alpha|-n}\left|D^{\alpha} u\right|^{2} d x \\
& +\sum_{|\alpha|=m}^{2 m} \gamma^{2 m} \int_{|x| \leq R}|x|^{-2 \tau+2|\alpha|-n+2 \epsilon}\left|D^{\alpha} u\right|^{2} d x \\
\leq & c \int_{|x|>R}|x|^{-2 \tau+4 m-n}\left|\left[\chi, \triangle^{m}\right] u\right|^{2} d x \\
\leq & c R^{-4 m} R^{-2 \tau+4 m-n}\|u\|_{H^{2 m}}^{2} \\
= & c\|u\|_{H^{2 m}}^{2} R^{-2 \tau-n},
\end{aligned}
$$

where $\|u\|_{H^{2 m}}^{2}$ is the $H^{2 m}$ norm of $u$ in the ball $B(0,2 R)$.

Recall that for $R=(\gamma \tau)^{-m / \epsilon}$, we have

$$
\begin{aligned}
\sum_{|\alpha| \leq 2 m} \int_{|x| \leq R / 2}\left|D^{\alpha} u\right|^{2} d x & \leq c R^{-4 m-4 \epsilon} 2^{-2 \gamma^{-1} R^{-\epsilon / m}}\|u\|_{H^{2 m}}^{2} \\
& \leq c e^{-\tilde{B} R^{-\epsilon / m}} .
\end{aligned}
$$

It should be noted that (2.7) is valid for $\tau \in \mathbb{N}+\frac{1}{2}$ and $R=(\gamma \tau)^{-m / \epsilon}$. Therefore, if we choose $\tau \in\left\{j+\frac{1}{2}: j \in \mathbb{N}\right\}$, then (2.7) only holds for $R_{j}=\left(\gamma\left(j+\frac{1}{2}\right)\right)^{-m / \epsilon}$. Nevertheless, we can see that

$$
R_{j+1}<R_{j}<2 R_{j+1} \quad \text { for } j \quad \text { large enough and } \quad R_{j} \rightarrow 0 \text { as } j \rightarrow \infty .
$$

Thus, we can conclude that

$$
\sum_{|\alpha| \leq 2 m} \int_{|x| \leq R}\left|D^{\alpha} u\right|^{2} d x \leq c e^{-B R^{-\epsilon / m}},
$$

for all sufficiently small $R>0$ with $B=2^{-2 \epsilon / m} \tilde{B}$.

\section{Carleman estimates With more singular Weights}

To prove the following Carleman estimates, we introduce polar coordinates in $\mathbb{R}^{n} \backslash\{0\}$ by setting $x=r \omega$, with $r=|x|, \omega=\left(\omega_{1}, \cdots, \omega_{n}\right) \in S^{n-1}$ when $x \neq 0$. Furthermore, setting $t=\log r$, we can substitute a new coordinate $t$ for $r$ such that

$$
\frac{\partial}{\partial x_{j}}=e^{-t}\left(\omega_{j} \partial_{t}+\Omega_{j}\right)
$$


where $\Omega_{j}$ is a vector field in $S^{n-1}$. Then the Laplacian becomes

$$
e^{2 t} \Delta=\partial_{t}^{2}+(n-2) \partial_{t}+\Delta_{\omega}
$$

where $\Delta_{\omega}=\sum_{j=1}^{n} \Omega_{j}^{2}$ is the Laplace-Beltrami operator in $S^{n-1}$. The vector field $\Omega_{j}$ has the properties

$$
\sum_{j=1}^{n} \omega_{j} \Omega_{j}=0, \quad \sum_{j=1}^{n} \Omega_{j} \omega_{j}=n-1 .
$$

The adjoint of $\Omega_{j}$ as an operator in $L^{2}\left(S^{2}\right)$ is

$$
\Omega_{j}^{*}=(n-1) \omega_{j}-\Omega_{j}
$$

and

$$
\sum_{j=1}^{n} \Omega_{j}^{*} \Omega_{j}=-\Delta_{\omega}
$$

Hereafter we shall use the following notation:

$$
\begin{aligned}
& D_{0}=(1 / i) \partial_{t} ; \\
& D_{k}=(1 / i) \Omega_{k}, \quad k=1, \cdots, n ; \\
& D^{\alpha}=D_{0}^{\alpha_{1}} \cdots D_{n}^{\alpha_{n}}, \quad \alpha=\left(\alpha_{0}, \cdots, \alpha_{n}\right) \in \mathbb{N}^{n+1} .
\end{aligned}
$$

When $r \rightarrow 0, t \rightarrow-\infty$, we will be interested in values of $t$ in a neighborhood of $-\infty$. Motivated by [13], we derive the following Carleman estimate with weights $\varphi_{\beta}=\varphi_{\beta}(x)=\exp \left(\frac{\beta}{2}(\log |x|)^{2}\right)$.

Theorem 3.1. Given $\sigma_{1} \in \mathbb{Z}$ and $\sigma_{2} \in \mathbb{Z}$, there exist a sufficiently large number $\beta_{0}>0$ and a sufficiently small number $r_{0}>0$ depending on $n, \sigma_{1}$ and $\sigma_{2}$ such that for all $u \in U_{r_{0}}$ with $0<r_{0}<e^{-1}, \beta \geq \beta_{0}$, we have that

$$
\begin{aligned}
& C \sum_{|\alpha| \leq 2} \beta^{3-2|\alpha|} \int \varphi_{\beta}^{2}|x|^{2 \sigma_{1}+2|\alpha|-n}(\log |x|)^{2 \sigma_{2}+2-2|\alpha|}\left|D^{\alpha} u\right|^{2} d x \\
& \leq \int \varphi_{\beta}^{2}|x|^{2 \sigma_{1}+4-n}(\log |x|)^{2 \sigma_{2}}|\Delta u|^{2} d x,
\end{aligned}
$$

where $U_{r_{0}}=\left\{u \in C_{0}^{\infty}\left(\mathbb{R}^{n} \backslash\{0\}\right): \operatorname{supp}(u) \subset B_{r_{0}}\right\}$ and $C$ is a positive constant.

Proof. By the polar coordinate system, we have

$$
\begin{aligned}
& \int \varphi_{\beta}^{2}|x|^{2 \sigma_{1}+4-n}(\log |x|)^{2 \sigma_{2}}|\Delta u|^{2} d x \\
= & \iint e^{\beta t^{2}} e^{2 \sigma_{1} t+4 t} t^{2 \sigma_{2}}|\Delta u|^{2} d t d \omega \\
= & \iint\left|e^{\beta t^{2} / 2} e^{\sigma_{1} t} t^{\sigma_{2}} e^{2 t} \Delta u\right|^{2} d t d \omega .
\end{aligned}
$$

If we set $u=e^{-\beta t^{2} / 2} e^{-\sigma_{1} t} t^{-\sigma_{2}} v$ and use (3.1), then

$$
e^{\beta t^{2} / 2} e^{\sigma_{1} t} t^{\sigma_{2}} e^{2 t} \Delta u=\left(\partial_{t}-\beta t\right)^{2} v+(n-2)\left(\partial_{t}-\beta t\right) v+\Delta_{\omega} v+a \partial_{t} v+b v,
$$

where $b=2 \beta \sigma_{1} t+2 \beta \sigma_{2}+\sigma_{1}^{2}+2 \sigma_{1} \sigma_{2} t^{-1}-\sigma_{2} t^{-2}+\sigma_{2}^{2} t^{-2}-(n-2)\left(\sigma_{1}+\sigma_{2} t^{-1}\right)$ and $a=-2 \sigma_{1}-2 \sigma_{2} t^{-1}$. 
By (3.6), (3.7) and $\beta$ large enough, (3.5) holds if we have

$$
\sum_{|\alpha| \leq 2} \beta^{3-2|\alpha|} \iint t^{2-2|\alpha|}\left|D^{\alpha} v\right|^{2} d t d \omega \leq C \iint\left|\Delta_{\beta} v\right|^{2} d t d \omega,
$$

where $\Delta_{\beta} v=\left(\partial_{t}-\beta t\right)^{2} v+(n-2)\left(\partial_{t}-\beta t\right) v+\Delta_{\omega} v$ and $C$ is a positive constant.

Note that

$$
\Delta_{\beta} v=\partial_{t}^{2} v+(n-2) \partial_{t} v-2 \beta t \partial_{t} v-(n-2) \beta t v-\beta v+\beta^{2} t^{2} v+\Delta_{\omega} v .
$$

Denote

$$
\Delta_{\beta}^{-} v=\partial_{t}^{2} v-(n-2) \partial_{t} v+2 \beta t \partial_{t} v-(n-2) \beta t v-\beta v+\beta^{2} t^{2} v+\Delta_{\omega} v .
$$

Using integration by parts, we get

$$
\begin{aligned}
I:= & \iint\left|\Delta_{\beta} v\right|^{2} d t d \omega-\iint\left|\Delta_{\beta}^{-} v\right|^{2} d t d \omega \\
= & \iint\left(12 \beta^{3} t^{2}+\beta^{2} O(t)\right)|v|^{2} d t d \omega+4 \beta \iint\left|\partial_{t} v\right|^{2} d t d \omega \\
& -4 \beta \iint \sum_{j}\left|\Omega_{j} v\right|^{2} d t d \omega .
\end{aligned}
$$

Similarly, we can get

$$
\begin{aligned}
J:= & \iint t^{-2}\left|\Delta_{\beta} v\right|^{2} d t d \omega+\iint t^{-2}\left|\Delta_{\beta}^{-} v\right|^{2} d t d \omega \\
= & \iint\left(2 \beta^{4} t^{2}+\beta^{3} O(t)\right)|v|^{2} d t d \omega+\int\left(8 \beta^{2}+\beta O\left(t^{-1}\right)\right)\left|\partial_{t} v\right|^{2} d t d \omega \\
& +2 \iint t^{-2}\left|\partial_{t}^{2} v\right|^{2} d t d \omega-\iint \sum_{j}\left(4 \beta^{2}+\beta O\left(t^{-1}\right)\right)\left|\Omega_{j} v\right|^{2} d t d \omega \\
& +2 \iint t^{-2}\left|\Delta_{\omega} v\right|^{2} d t d \omega+4 \iint t^{-2} \sum_{j}\left|\partial_{t} \Omega_{j} v\right|^{2} d t d \omega
\end{aligned}
$$

If $v \in C_{0}^{\infty}\left(B\left(0, e^{T_{0}}\right)\right)$ and $\beta>>\left|T_{0}\right|$ is large enough, then it follows from (3.9) and (3.10) that

$$
\begin{aligned}
\beta I+J \geq & 13 \beta^{4} \iint t^{2}|v|^{2} d t d \omega+2 \iint t^{-2}\left|\Delta_{\omega} v\right|^{2} d t d \omega \\
& -9 \beta^{2} \iint \sum_{j}\left|\Omega_{j} v\right|^{2} d t d \omega+11 \beta^{2} \iint\left|\partial_{t} v\right|^{2} d t d \omega \\
& +2 \iint t^{-2}\left|\partial_{t}^{2} v\right|^{2} d t d \omega+4 \iint t^{-2} \sum_{j}\left|\partial_{t} \Omega_{j} v\right|^{2} d t d \omega \\
\geq & K+\frac{13}{39} \beta^{4} \iint t^{2}|v|^{2} d t d \omega+\frac{1}{20} \iint t^{-2}\left|\Delta_{\omega} v\right|^{2} d t d \omega \\
& +\beta^{2} \iint \sum_{j}\left|\Omega_{j} v\right|^{2} d t d \omega+11 \beta^{2} \iint\left|\partial_{t} v\right|^{2} d t d \omega \\
& +2 \iint t^{-2}\left|\partial_{t}^{2} v\right|^{2} d t d \omega+4 \iint t^{-2} \sum_{j}\left|\partial_{t} \Omega_{j} v\right|^{2} d t d \omega
\end{aligned}
$$


where

$$
K=\frac{500}{39} \beta^{4} \iint t^{2}|v|^{2} d t d \omega-10 \beta^{2} \iint \sum_{j}\left|\Omega_{j} v\right|^{2} d t d \omega+\frac{39}{20} \iint t^{-2}\left|\Delta_{\omega} v\right|^{2} d t d \omega .
$$

Now we shall get a lower bound of $K$. Then, by (3.3), we have

$$
-\beta^{2} \iint \sum_{j}\left|\Omega_{j} v\right|^{2} d t d \omega=\beta^{2} \iint \bar{v} \Delta_{\omega} v d t d \omega .
$$

Since $10|a b| \leq 500 a^{2} / 39+39 b^{2} / 20$, it follows that

$$
10\left|\beta^{2} v \Delta_{\omega} v\right| \leq \frac{500}{39} \beta^{4} t^{2}|v|^{2}+\frac{39}{20} t^{-2}\left|\Delta_{\omega} v\right|^{2} .
$$

Combining (3.12) and (3.13), we get a lower bound of $K$ :

$$
\begin{aligned}
K & =\frac{500}{39} \beta^{4} \iint t^{2}|v|^{2} d t d \omega-10 \beta^{2} \iint \sum_{j}\left|\Omega_{j} v\right|^{2} d t d \omega+\frac{39}{20} \iint t^{-2}\left|\Delta_{\omega} v\right|^{2} d t d \omega \\
& \geq 0 .
\end{aligned}
$$

In addition, the ellipticity of $\Delta_{\omega}$ implies that there exists a new positive constant $C$ such that

$$
\sum_{|\alpha|=2} \iint t^{-2}\left|\Omega^{\alpha} v\right|^{2} d t d \omega \leq C \iint t^{-2}\left|\Delta_{\omega} v\right|^{2} d t d \omega
$$

Thus, it follows from (3.11), (3.14) and (3.15) that

$$
\begin{aligned}
C(\beta I+J) \geq & \beta^{4} \iint t^{2}|v|^{2} d t d \omega+\iint t^{-2}\left|\Delta_{\omega} v\right|^{2} d t d \omega \\
& +\beta^{2} \iint \sum_{j}\left|\Omega_{j} v\right|^{2} d t d \omega+\beta^{2} \iint\left|\partial_{t} v\right|^{2} d t d \omega \\
& +\iint t^{-2}\left|\partial_{t}^{2} v\right|^{2} d t d \omega+\iint t^{-2} \sum_{j}\left|\partial_{t} \Omega_{j} v\right|^{2} d t d \omega
\end{aligned}
$$

Together with

$$
\beta I+J \leq(\beta+1) \iint\left|\Delta_{\beta} v\right|^{2} d t d \omega
$$

(3.8) holds. So we have the result.

Corollary 3.1. Given $\sigma_{1} \in \mathbb{Z}$ and $\sigma_{2} \in \mathbb{Z}$, there exist a sufficiently large number $\beta_{0}>0$ and a sufficiently small number $r_{0}>0$ depending on $n, m, \sigma_{1}$ and $\sigma_{2}$ such that for all $u \in U_{r_{0}}$ with $0<r_{0}<e^{-1}, \beta \geq \beta_{0}$, we have that

$$
\begin{aligned}
& C \sum_{|\alpha| \leq 2 m} \beta^{3 m-2|\alpha|} \int \varphi_{\beta}^{2}|x|^{2 \sigma_{1}+2|\alpha|-n}(\log |x|)^{2 \sigma_{2}+2 m-2|\alpha|}\left|D^{\alpha} u\right|^{2} d x \\
& \leq \int \varphi_{\beta}^{2}|x|^{2 \sigma_{1}+4 m-n}(\log |x|)^{2 \sigma_{2}}\left|\Delta^{m} u\right|^{2} d x
\end{aligned}
$$

where $C$ is a positive constant. 
Corollary 3.2. There exist a sufficiently large number $\beta_{0}>0$ and a sufficiently small number $r_{0}>0$ depending on $n$ and $m$ such that for all $u \in U_{r_{0}}$ with $0<r_{0}<$ $e^{-1}, \beta \geq \beta_{0}$, we have that

$$
\begin{aligned}
& C \sum_{|\alpha| \leq 2 m} \beta^{3 m-2|\alpha|} \int \varphi_{\beta}^{2}|x|^{2|\alpha|-n}(\log |x|)^{2 m-2|\alpha|}\left|D^{\alpha} u\right|^{2} d x \\
& \leq \int \varphi_{\beta}^{2}|x|^{4 m-n}\left|\Delta^{m} u\right|^{2} d x
\end{aligned}
$$

where $C$ is a positive constant.

Remark 3.2. The estimate (3.16) in Corollary 3.2 remains valid if we assume $u \in$ $H_{l o c}^{2 m}(\Omega)$ with compact support and satisfies for all $|\alpha| \leq 2 m, \int_{|x| \leq R}\left|D^{\alpha} u\right|^{2} d x=$ $O\left(e^{-B R^{-\epsilon / m}}\right)$ as $R \rightarrow 0, B>0$.

\section{Proof of Theorem 1.1}

Let $u \in H_{l o c}^{2 m}(\Omega)$ be a solution of (1.2). By Lemma 2.1 and Theorem 2.3 $u$ is in $H_{l o c}^{2 m}(\Omega)$ and satisfies (2.3). Thus by Remark 3.2 we can apply (3.16) with the function $\xi u$, where $\xi(x) \in C_{0}^{\infty}\left(\mathbb{R}^{n}\right)$ such that $\xi(x)=1$ for $|x| \leq R$ and $\xi(x)=0$ for $|x| \geq 2 R$ ( $R>0$ small enough). Then

$$
\begin{aligned}
& \sum_{|\alpha| \leq\left[\frac{3 m}{2}\right]} \beta^{3 m-2|\alpha|} \int_{|x|<R} \varphi_{\beta}^{2}|x|^{2|\alpha|-n}(\log |x|)^{2 m-2|\alpha|}\left|D^{\alpha} u\right|^{2} d x \\
\leq & \sum_{|\alpha| \leq 2 m} \beta^{3 m-2|\alpha|} \int_{|x|<R} \varphi_{\beta}^{2}|x|^{2|\alpha|-n}(\log |x|)^{2 m-2|\alpha|}\left|D^{\alpha} u\right|^{2} d x \\
\leq & \sum_{|\alpha| \leq 2 m} \beta^{3 m-2|\alpha|} \int \varphi_{\beta}^{2}|x|^{2|\alpha|-n}(\log |x|)^{2 m-2|\alpha|}\left|D^{\alpha}(\xi u)\right|^{2} d x \\
\leq & c \int_{\varphi_{\beta}^{2}|x|^{4 m-n}\left|\Delta^{m}(\xi u)\right|^{2} d x} \\
\leq & c \int_{|x| \leq R} \varphi_{\beta}^{2}|x|^{4 m-n}\left|\triangle^{m} u\right|^{2} d x+c \int_{|x|>R} \varphi_{\beta}^{2}|x|^{4 m-n}\left|\left[\xi, \triangle^{m}\right] u\right|^{2} d x \\
\leq & c \sum_{|\alpha| \leq m-1} \int_{|x| \leq R} \varphi_{\beta}^{2}|x|^{2|\alpha|-n}\left|D^{\alpha} u\right|^{2} d x \\
& +c \sum_{|\alpha|=m}^{[3 m / 2]} \int_{|x| \leq R} \varphi_{\beta}^{2}|x|^{2|\alpha|-n+2 \epsilon}\left|D^{\alpha} u\right|^{2} d x \\
& +c \int_{|x|>R} \varphi_{\beta}^{2}|x|^{4 m-n}\left|\left[\xi, \triangle^{m}\right] u\right|^{2} d x,
\end{aligned}
$$

because $\lim _{|x|=r \rightarrow 0} r^{2 \epsilon}(\log r)^{-2 m}=0$ which implies $c|x|^{2 \epsilon} \leq(\log |x|)^{2 m}$ as $R \leq R_{0}$ for some small $R_{0}>0$. Let $\beta>\beta_{0}$ ( $\beta_{0}$ large enough); the first two terms on the right-hand side will be swallowed by the left-hand side. Recall that $\varphi_{\beta}=\varphi_{\beta}(x)=$ 
$\exp \left(\frac{\beta}{2}(\log |x|)^{2}\right)$. Therefore, we obtain that

$$
\begin{aligned}
& \beta^{3 m} \exp \left(\beta\left(\log R_{0}\right)^{2}\right) \int_{|x|<R_{0}}|x|^{-n}(\log |x|)^{2 m}|u|^{2} d x \\
\leq & \sum_{|\alpha| \leq\left[\frac{3 m}{2}\right]} \beta^{3 m-2|\alpha|} \int_{|x|<R_{0}} \varphi_{\beta}^{2}|x|^{2|\alpha|-n}(\log |x|)^{2 m-2|\alpha|}\left|D^{\alpha} u\right|^{2} d x \\
\leq & c \int_{|x|>R_{0}} \varphi_{\beta}^{2}|x|^{4 m-n}\left|\left[\xi, \triangle^{m}\right] u\right|^{2} d x \\
\leq & c \exp \left(\beta\left(\log R_{0}\right)^{2}\right) \int_{|x|>R_{0}}|x|^{4 m-n}\left|\left[\xi, \triangle^{m}\right] u\right|^{2} d x .
\end{aligned}
$$

Divide $\exp \left(\beta\left(\log R_{0}\right)^{2}\right)$ into both sides of (4.2) and then let $\beta \rightarrow \infty$; this implies that $u=0$ in $B\left(0, R_{0}\right)$. By standard arguments, we can get $u=0$ in $\Omega$. This completes the proof.

\section{ACKNOWLEDGEMENT}

I would like to thank Professor Jenn-Nan Wang for his many constructive suggestions and comments.

\section{REFERENCES}

1. S. Alinhac and M.S. Baouendi, A counterexample to strong uniqueness for the partial differential equations of Schrödinger's type, Comm. in P.D.E. 19 (1994), 1723-1733. MR1294476 (95g:35043)

2. N. Aronszajn, A. Krzywicki and J.Szarski, A unique continuation theorem for exterior differential forms on Riemannian manifolds, Ark. Mat. 4 (1962), 417-453. MR0140031 (25:3455)

3. B. Barcelo, C.E. Kenig, A. Ruiz and C.D. Sogge, Weighted Sobolev inequalities for the Laplacian plus lower order terms, Illinois J. Math. 32 (1988), 230-245. MR0945861 (89h:35048)

4. P. Le Borgne, Strong uniqueness for fourth order elliptic differential operators, Indiana Univ. Math. J. 50, No. 1, (2001), 353-381. MR.1857040(2003i:35064)

5. C. Grammatico, A result on strong unique continuation for the Laplace operator, Comm. in P.D.E. 22 (1997), 1475-1491. MR1469579 (98j:35034)

6. F. Colombini and C. Grammatico, Some remarks on strong unique continuation for the Laplace operator and its power, Comm. in P.D.E. 24 (1999), 1079-1094. MR.1680873 (2000c:35263)

7. F. Colombini and C. Grammatico, A counterexample to strong unique continuation for all powers of the Laplace operator, Comm. in P.D.E. 25 (2000), 586-600. MR 1748356 (2001c:35067)

8. L. Hörmander, Uniqueness theorems for second order elliptic differential equations, Comm. in P.D.E. 8, No. 1, (1983), 21-64. MR0686819 (85c:35018)

9. L. Hörmander, "The analysis of linear partial differential operators", Vol. 3, Springer-Verlag, Berlin, New York, 1985. MR0781536 (87d:35002a)

10. T. Ōkaji, Uniqueness of the Cauchy problem for elliptic operators with fourfold characteristics of constant multiplicity, Comm. in P.D.E. 22 (1997), 269-290. MR1434146 (97k:35004)

11. Y. Pan, Unique continuation for Schrödinger operators with singular potentials, Comm. in P.D.E. 17 (1992), 953-965. MR 1177300 (93j:35064)

12. M.H. Protter, Unique continuation for elliptic equations, Trans. Amer. Math. Soc. 95 (1960), 81-91. MR0113030 (22:3871)

13. R. Regbaoui, Strong uniqueness for second order differential operators, J. Diff. Eq. 141 (1997), 201-217. MR 1488350 (98i:35002)

14. K. Watanabe, On the uniqueness of the Cauchy problem for certain elliptic equations with triple characteristics, Tohoku Math. J. 23 (1971), 473-490. MR0308584 (46:7698) 
15. T.H. Wolff, "Fourier analysis and partial differential equations", Miraflores de la Sierra (1992), 99-128. MR1330234 (96c:35068)

16. T.H. Wolff, A counterexample in a unique continuation problem, Comm. Anal. Geom. 2 (1994), 79-102. MR1312679 (96a:35018)

Department of Mathematics, National Chung-Cheng University, Chia-Yi 62117, TAIWAN

E-mail address: cllin@math.ccu.edu.tw 